\section{Morbus Fabry - das Chamäleon wird häufig übersehen}

\begin{abstract}
Auf dem heartlive in Stuttgart wurde auch über eine Multisystemerkrankung gesprochen, die vorrangig Herz, Niere und Gehirn betrifft. Die Rede ist vom Morbus Fabry, einer vererbten lysosomalen Speicherkrankheit. Als Kardiologe sollten Sie insbesondere bei unklarer linksventrikulärer Hypertrophie an diese Erkrankung denken; denn eine frühzeitige Therapie verbessert nicht nur die Lebensqualität, sondern auch die Prognose.
\end{abstract}

$\mathrm{D}$ as ist doch ein Kolibri, den ich in meiner Praxis wohl kaum erleben werde! - so lautet die weit verbreitete Meinung unter Ärzten, wenn sie bei Fortbildungsveranstaltungen mit dem Morbus Fabry konfrontiert werden. „Deshalb wird an dieses Krankheitsbild viel zu selten gedacht", sagte Dr. Martin Steeg von der kardiologischen Universitätsklinik in Tübingen.

Betroffene Patienten hätten meist eine lange Odyssee mit Fehldiagnosen hinter sich, bevor im Durchschnitt nach zehn Jahren die richtige Diagnose gestellt werde. Doch eine frühzeitige Diagnosestellung und Therapie ist wichtig und zwar nicht nur wegen der starken Beschwerden, sondern auch im Hinblick auf die Lebenserwartung.

„Unbehandelt ist die Lebenserwartung betroffener Patienten um 20 Jahre verkürzt", betonte Steeg.

\section{Vererbte lysosomale Speicherkrankheit}

Beim M. Fabry handelt es sich um eine lysosomale Speicherkrankheit, die Xchromosomal vererbt wird und deshalb vorwiegend bei Männern auftritt, wobei das mittlere Manifestationsalter zwischen drei und zehn Jahren liegt. Bei heterozygoten Frauen verläuft die Erkrankung meist mild, aber es gibt auch schwere Verlaufsformen. Der genetisch bedingte Mangel des Enzyms alpha-Galaktosidase A führt zu einer progredienten Akkumulation des Sphingolipids Globotriaosylceramid in den Zellen, was zu irreversiblen Schäden in multiplen Organsystemen insbesondere Herz, Niere und Gehirn führen kann.

\section{Klinische Symptomatik} variiert je nach Alter

Die klinische Symptomatik variiert je nach Alter der Patienten. Bei Kindern und Jugendlichen stehen neuropathische Schmerzen im Vordergrund. Dazu kommen multiple Angiome und ophthalmologische Auffälligkeiten nämlich die Cornea verticillata. Typisch ist auch eine Hypohidrose und eine Kälte- bzw. Hitze-Überempfindlichkeit. Aber auch eine renale Beteiligung in Form einer Proteinurie kann bereits bei Kindern auftreten.

Wie ein Chamäleon lässt sich der Morbus Fabry wegen seines symptomatischen Facettenreichtums nur schwer entdecken.
Bei Erwachsenen kommt es zu einer zunehmenden Nephropathie mit Proteinurie und fortschreitender Niereninsuffizienz sowie einer hypertrophen Kardiomyopathie. Die Nephropathie verläuft rasch progredient mit einer Abnahme der glomerulären Filtrationsrate (GFR) um 10 bis $15 \mathrm{ml} / \mathrm{min}$ pro Jahr.

„Typisch für die Fabry-Kardiomyopathie ist die konzentrische linksventrikuläre Hypertrophie ohne Obstruktion des Ausflusstraktes mit prominenten Papillarmuskeln“, erklärte Steeg. Dabei komme es auch zu einer intramyokardialen Fibrosierung. Die Folge ist eine diastolische Herzinsuffizienz. Betroffen ist auch das Reizleitungssystem, was zu AV-Blockierungen, aber auch zu ventrikulären Arrhythmien bis hin zum akuten Herztod und Vorhofflimmern führen kann. „Bei jeder unklaren Hypertrophie sollten Sie an einen M. Fabry denken“, so Steeg.

In fortgeschrittenen Stadien ist auch das Gehirn betroffen. Im MRT zeigen sich in diesem Falle „white matter lesions“, vergleichbar mit einer Vaskulitis. Klinische Manifestationen sind transiente ischämische Attacken (TIA) und Insulte. Von Seiten des Magen-DarmTraktes können neben krampfartigen Schmerzen auch Durchfälle oder Obstipation auftreten.

\section{Einfache Diagnostik durch Bestimmung der Enzymaktivität}

Für den Nachweis oder Ausschluss der Erkrankung genügt eine TrockenblutUntersuchung zur Bestimmung der Enzymaktivität der alpha-Galaktosidase A. Bei Frauen gelingt der Nachweis der Erkrankung häufig nur mithilfe eines Gentests.

„Entscheidend für die Prognose der Patienten ist ein möglichst frühzeitiger Beginn der kausalen Enzymersatztherapie“, darauf wies Dr. Markus Cybulla, Rheumatologe und Nephrologe in Müllheim im Breisgau, hin. Dadurch könne der Krankheitsverlauf sowohl an der Niere als auch am Herzen aufgehalten werden. Die Therapie führe zu einer Abnahme der linksventrikulären Masse, zu einer Stabilisierung der Nierenfunktion, zu einer Verringerung der Nervenschmerzen und somit auch zu einer Verbesserung der Lebensqualität.

Dr. med. Peter Stiefelhagen 